\title{
Ruptured aneurysm of the noncoronary sinus of Valsalva into the right atrium
}

\author{
Diana Rudan*, Stanko Biočić, Željko Đurašević, Josip Vincelj \\ University Hospital Dubrava, Zagreb, Croatia
}

\begin{abstract}
Aneurysms of sinus of Vasalva are rare cardiac abnormalities, with an incidence of less than $0.15 \%$, and marked male predominance. ${ }^{1}$ They are dilatations of the aortic sinuses, and are classified as congenital or acquired. The congenital aneurysms are more common and often caused by weakness at the junction of the aortic media and the annulus fibrosus. They commonly coexist with other malformations such as ventricular septal defect, anomalies of the aortic valve and coarctaton of the aorta. Acquired aneurysms are caused by conditions affectng the aortic wall, such as infections, trauma, or connective tissue disorders. It may be asymptomatic, or it may present as angina, or with symptoms of valvular insufficiency. Once ruptured, it often produces hemodynamic instability. The frequency of ruptured sinus Valsalva aneurysm varies according to the location: $60 \%$ are located in the right sinus, $42 \%$ in the noncoronary sinus and $10 \%$ in the left sinus. ${ }^{2}$ According to the location and frequency, rupture of a sinus Valsalva aneurysm occurs principally in the right ventricle $(60 \%)$ or in the right atrium $(29 \%)$. Extracardiac ruptures are rare. When rupture is suspected, immediate diagnosis should be pursued with transthoracic echocardography.
\end{abstract}

We present a case of 33-year-old man admitted to our emergency departament with symptoms of abdominal pain, abnormal liver function tests and new diagnosed heart murmur. On physical examination his blood pressure was $120 / 60 \mathrm{mmHg}$ and heart rate of $95 \mathrm{bpm}$. Jugular venous distension was present. There were no pulmonic rales or signs of peripheral edema. On auscultation, there was continuous systolic-dyastolic murmor of grade III/IV, best heard at the apex. Chest X-ray showed cardiomegaly. Transthoracic echocardography showed dilatation of the left ventricle, reduced systolic function, dilatation of the right ventricle and left atrium, and noncoronary sinus Valsalva aneurysm ruptured in the right atrium. The aortic valve was three-leaflet, and had no insufficiency, no ventricular septal defecte was present. The patient underwent corrective surgery, confirming the aneurysm of noncoronary sinus valsava ruptured in the right cardiac chambers. The patient has been followed-up in our cardiology clinic with complete recovery.

KEYWORDS: aneurysm of sinus Valsava, echocardiography, corrective surgery.

\section{Literature}

1. Lakoumentas JA, Bonou MS, Brili S, Theocaharis CS, Benroubis AD, Perpinia AS, et al. Ruptured aneurysm of the right sinus of Valsava into the right ventricule. Hellenic J Cardiol. 2002;43:242-5.

2. Galicia-Tornell MM, Marin-Solis B, Mercado-Astorga O, Espinosa-Anguiano S, Martinez-Martinez M, Villalpando-Mendoza E. Sinus of Valsava aneurysm with rupture. Case report and literature review. Cir Cir. 2009;77:441-5. 